\title{
Covalent quantum dot receptor linkage via the acyl carrier protein for single-molecule tracking, internalization, and trafficking studies
}

\author{
Monika Zelman-Femiak ${ }^{1}$, Kun Wang ${ }^{1}$, Kira V. Gromova ${ }^{1, *}$, Petra Knaus ${ }^{2}$, and Gregory S. Harms $s^{1,3}$ \\ ${ }^{1}$ Bio-Imaging Center, RudolfVirchow Center, University of Würzburg, Würzburg, Germany, ${ }^{2}$ Institute for \\ Chemistry and Biochemistry, Free University of Berlin, Berlin, Germany, and ${ }^{3}$ Department of Biology and Physics, \\ Wilkes University, Wilkes-Barre, Pennsylvania, USA
}

BioTechniques 49:574-579 (August 2010) doi 10.2144/000113466

Key words: quantum dot; single-molecule tracking; BMP; PTH; ACP

Supplementary material for this article is available at www.BioTechniques.com/article/113466.

*K.V.G.s present address is the Institute for Chemistry and Biochemistry, Free University of Berlin, Takustr. 6, 14195 Berlin, Germany.

Here we describe a labeling technique for the covalent linkage of quantum dots to transmembrane receptors for single-molecule tracking. Our method combines the acyl carrier protein (ACP) technique with coenzyme A (CoA)-functionalized quantum dots to covalently attach quantum dots to ACP fusions of receptor proteins. The advantages of this approach include: (i) the use of a smaller attachment linker than in many other quantum dot-labeling systems; (ii) the ability to achieve a reliable 1:1 fluorophore-to-receptor labeling stoichiometry; (iii) the specificity of the method; and (iv) the covalent nature of the quantum dot linkage. We demonstrate the general suitability of this technique in single-molecule tracking, internalization, and trafficking studies by imaging two different transmembrane receptors in living cells.

Detection of single fluorescently labeled proteins is useful in determining their local mobility, local stoichiometry, fundamental molecular interactions and molecular function. Numerous single-molecule studies have demonstrated the unique functions of proteins, revealing both large and subtle details of their biomolecular interactions. Two key issues with such studies are the biochemical method of fluorophore attachment and the proper choice and use of the fluorophore itself (for reviews, see References 1-4).

For analysis of the molecular mobility of membrane receptors, specific localization along with the correct determination of the motion of the fluorescently labeled receptor requires high signal-to-background ratios and acquisition rates and a rigid attachment of a minimized fluorophore $(5,6)$. Fluorescently labeled receptors and ion channels for single-molecule studies on living cells can be obtained with fluorescent fusion proteins (7-10), by direct covalent bonding of fluorophores to cysteine or amino reactive groups, or with protein tags that react with modified fluorophores (11). However, while these techniques are well-established for standard organic fluorophores, general schemes for direct labeling by quantum dots (QDs) are still in their infancy (1-4).
Single-molecule tracking with QDs displays superior performance over tracking with large latex beads since QDs are $\sim 10 \times$ smaller. In addition, they typically yield higher fluorescence signals than standard organic dyes and fluorescent proteins due to their improved brightness and resistance to photobleaching. With the recent advent of small, noncommercially available QDs, the size limitations of standard commercial QDs have been overcome, making them comparable in size to fluorescent proteins and only somewhat larger than the brightest fluorescing organic dyes $(12,13)$.

QD tracking has been limited in the number of available labeling techniques. To date, most QDs for tracking are conjugated to antibodies, which unless they are directly conjugated to primary $\mathrm{F}_{\mathrm{ab}}$ fragments, can increase the effective radius of the QD by $\geq 10 \mathrm{~nm}(1,3,4)$. Although tracking receptors with QD-conjugated secondary antibodies has been successful $(1,3)$, the desire to reduce the common drawbacks of antibody labeling (14-17) has led to new direct QD binding techniques.

Direct attachment of QDs to receptors can be achieved by normal amino or cysteine nonspecific reactions (1-4). Another possibility is the biotinylation of the receptor and addition of either streptavidin-coated QDs or QDs conjugated with the recently generated $\mathrm{m}$-streptavidin of only 159 amino acid residues $(18,19)$. Yet another method is to covalently link a QD to a fusion protein containing a HaloTag, a modified haloalkane dehydrogensase of 296 amino acids, which can covalently bind a modified chloroalkanelabeled QD (20,21).

Many other labeling systems are useful for imaging and biological quantification of proteins (22-24); however, with the exception of the aformentioned techniques, they are either noncovalent or lack suitable protocols for QD labeling. Some systems —including FlAsH (25), hexahistidine (13,26,27), biotin acceptor peptide (28), and Q-tag (29) - are so stable with organic dyes that they have been demonstrated with single-molecule detection.

The acyl carrier protein (ACP) system is especially well-suited for the specific labeling and tracking of single receptors on living cells (30). With only 77 amino acids, ACP is relatively smaller than comparable tags such as the SNAP and CLIC tags (22-24) and has reliable reactivity to coenzyme A (CoA) bound to organic fluorophores or to biotin (30) in a reaction catalyzed by phosphopantetheinyl transferase (PPTase). 

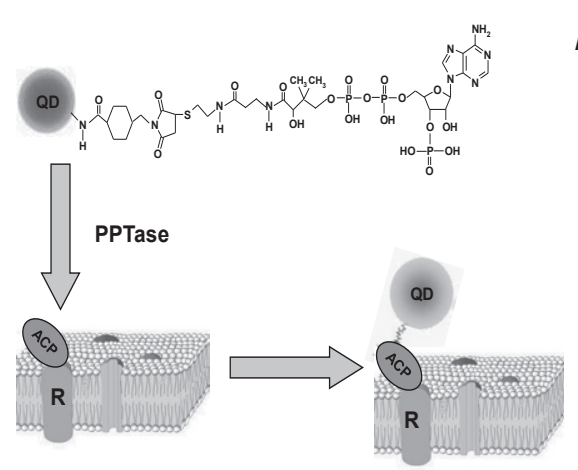

Figure 1. Schematic diagram of labeling technique. By application of the enzyme PPTase, a single $\mathrm{QD}$ functionalized to CoA is transferred to the ACP protein fused to the receptor.

The advantages of tracking with ACP-CoA and organic fluorophores were demonstrated on $\mathrm{G}$ protein-coupled receptors (GPCRs) (9,10), and AMPA receptors (31). In particular, tracking with $\mathrm{ACP}$ is advantageous since the receptors fused to ACP retain biological activity. Furthermore, the covalent binding of the CoA fluorophore by ACP is specific and efficient. The same system was also used for internalization studies $(9,10,31)$. However, until now, protocols for the direct attachment of QDs to receptors via an ACP tag did not exist.

Here we introduce a method for labeling ACP tags with QDs covalently bound to CoA in a 1:1 ratio. The QD:CoA covalent binding occurs via straightforward thiol-reactive chemistry in an appropriate concentration of amine-blocking groups on the QDs. We demonstrate the successful labeling of two different receptors: the parathyroid hormone receptor (PTHR), which is a GPCR, and the bone morphogenetic protein type II receptor (BRII). We compare the labeling and tracking of both receptors with either our ACP-CoAQD technique or antibody QD conjugates. We find that ACP-CoA-QD labeling enables more precise tracking, longer tracking times, and the possibility to determine all stages in the fate of individual receptors, including behavior at the membrane, internalization, and postinternalization trafficking events, such as recycling. To our knowledge, this technique provides the shortest specific covalent tether of a $\mathrm{QD}$ with a receptor.

\section{Materials and methods}

\section{Plasmid construction of ACP-}

\section{PTHR-TC and ACP-BRII-TC1}

The ACP fusion to the $\mathrm{N}$ terminus of the truncated human PTHR was produced in a similar manner as previously reported for the N-terminally labeled GFP-PTHR (32). The PCR products of the $\mathrm{N}$-terminal signal peptide of PTHR, ACP, and of the human PTHR truncated to amino acids 102-270
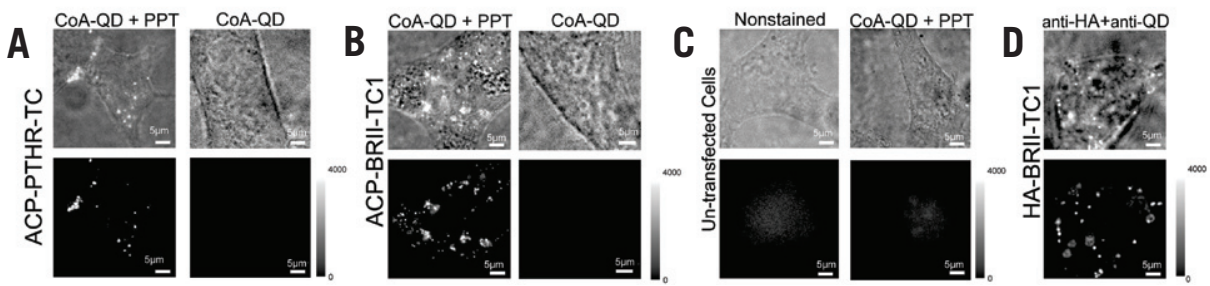

Figure 2. Characterization of the ACP-COA-QD labeling of receptors on living cells. In each panel, fluorescent images are shown at the bottom, while the corresponding transmission image is shown above. (A) CoA-QD labeling of HEK293 cells transfected with ACP-PTHR-TC followed by PPTase treatment shows many QDs (bottom left). Non-PPTase-treated cells show no QDs (bottom right). (B) CoA-QD labeling of HEK293 cells transfected with the ACP-BRII-TC1 plasmid followed by PPTase shows many QDs (bottom left), while without PPTase treatment, there are no QDs (bottom right). (C) Negative control of nontransfected HEK293 cells without (left) and with (right) CoA-QD and PPTase treatment. (D) Antibody-QD labeling of transfected HEK293 cells (transmission image, top) with the HA-BRII-TC1 plasmid with both the primary anti-HA antibody and secondary $F_{a b}$ antibody QD conjugate showing many QDs (bottom). All intensity scaling is in photons $/ 5 \mathrm{~ms}$.

were subcloned together by ligation after digestion with the same restriction enzyme into pcDNA3 (Invitrogen, Karlsruhe, Germany) for transfection and expression in mammalian cells. The final construct did not include the pharmacologically unimportant residues 61-101 but did include a two-residue linker (from restriction sites) between the ACP C terminus and the truncated PTHR $\mathrm{N}$ terminus and a six-residue linker between the ACP N terminus and the signal peptide of PTHR.

The ACP fusion to the $\mathrm{N}$ terminus of the truncated type II human bone morphogenetic receptor (BRII-TC1) was produced in a similar manner as above following similar strategies $(33,34)$. The final construct also included a six-residue linker between the ACP $\mathrm{C}$ terminus and the BRII-TC1 $\mathrm{N}$ terminus. All constructs, which were subcloned into pcDNA3, were verified by sequencing.

\section{Cell culture}

Human embryonal kidney cells (HEK293) were cultured in DMEM (Sigma-Aldrich, Taufkirchen, Germany) supplemented with 10\% FCS (PAA Laboratories GmbH, Linz, Austria) and 1\% penicillin/streptomycin (Sigma-Aldrich) solution at $37^{\circ} \mathrm{C} / 5 \% \mathrm{CO}_{2}$. FuGENE 6 (Roche Applied Science, Indianapolis, IN, USA). Effectene (Qiagen, Hilden, Germany) transfection reagents were used for HEK293 cell transient transfections. For single-molecule imaging, cells were seeded into six-well plates containing glass coverslips (No. 1, round, $24 \mathrm{~mm}$; Assistent, Sondheim, Germany) and transfected with $0.2 \mu \mathrm{g}$ plasmid DNA per well.

\section{Labeling}

Labeling was performed by incubating the cells on the coverslips for 15-20 min at $37^{\circ} \mathrm{C}$ in $270 \mu \mathrm{L}$ DMEM with $1 \%$ BSA (Sigma-Aldrich), $1.5 \mu \mathrm{M} 6 \times$ His-PPTase, and $0.3 \mathrm{nM} \mathrm{CdSe} / \mathrm{ZnS}$ QD-CoA molecules prepared with the QD655 Qdot Antibody Conjugation Kit (NrQ22021MP; Molecular
Probes, Invitrogen Detection Technologies, Eugene, Oregon, USA; Invitrogen, Karlsruhe, Germany) and reacted with CoA (SigmaAldrich) (for details of the reaction for a 1:1 QD:CoA ratio, see the Supplementary Materials). Samples were washed three times with $500 \mu \mathrm{L}$ DMEM before measurements. Experiments were conducted on cells in 300 $\mu \mathrm{L}$ DMEM.

\section{Ligands}

Human PTH peptide ligand hPTH (amino acids 1-34) purchased from Bachem California (Torrance, CA, USA) was used to bind the truncated $2.5 \mathrm{nM}$ PTHR. Human bone morphogenetic protein-2 (BMP-2; Sigma-Aldrich) was used to bind the truncated BRII-TC1. Ligands diluted in PBS were added in concentrations from $1 \mathrm{nM}$ to $10 \mathrm{nM}$, depending on the experiment.

\section{Single-molecule microscopy}

The experimental arrangement for singlemolecule imaging has been described in detail previously $(35,36)$. Essentially, the samples were mounted onto an inverted microscope (Axiovert 200; Zeiss, Göttingen, Germany) equipped with a $100 \times$ objective (PlanNeofluor $100 \times$, N.A. = 1.3; Zeiss), and illuminated for $5 \mathrm{~ms}$ with the 473-nm laser line of a diode laser (MBL-473-100; CNI Laser; Changchun, China) with excitation intensity of $0.5-1 \mathrm{~kW} / \mathrm{cm}^{2}$ and synchronized with the exposure of the Peltier-cooled electron-multiplying charge-coupled device (EMCCD) camera system (CascadeII 512B; Roper Scientific, Tucson, AZ, USA).

\section{Total internal reflection} single-molecule microscopy The total internal reflection (TIRF) single-QD imaging and tracking were performed on a Leica AM TIRF system (Leica, Wetzlar, Germany). The TIRF microscope was customequipped from the manufacturer with a $100 x$, N.A. $=1.46$ objective (Leica), a $405-\mathrm{nm}$, 50-mW diode laser (CUBE; Coherent, Santa 
A
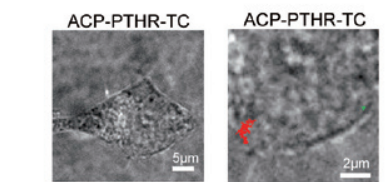

4000
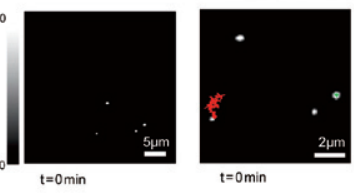

C

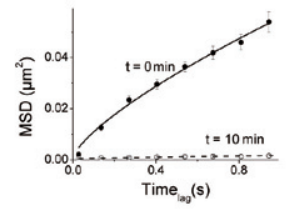

E

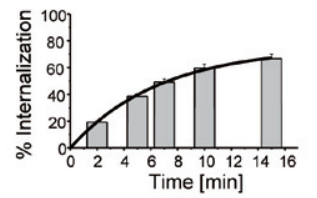

B

ia. $200 \mathrm{~nm}$

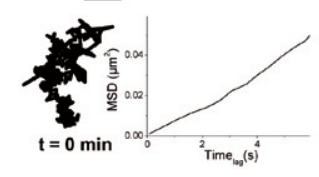

ib. $200 \mathrm{~nm}$

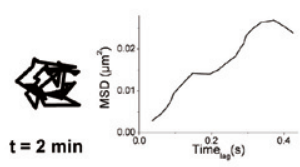

iia.

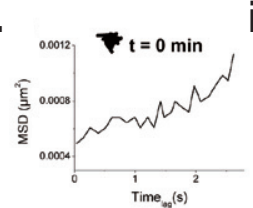

iib.

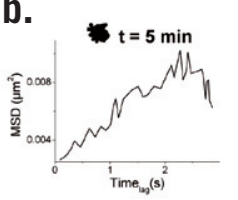

iic.

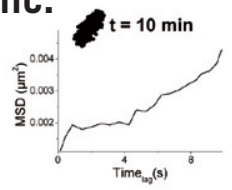

D
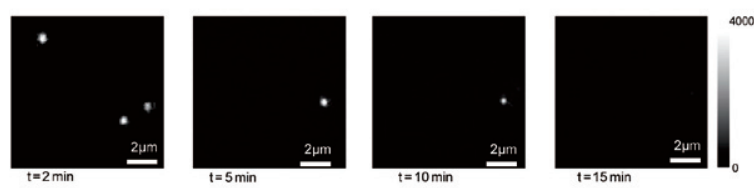

F
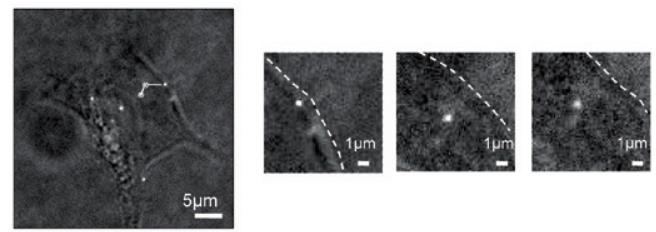

Figure 3. Tracking of the surface mobility and internalization of ACP-PTHR-TC in live HEK293 cells. (A) Transmission (upper) and TIRF images (lower) of the CoA-QD-labeled ACP-PTHR-TC on HEK293 cells (left). Two traces in a magnified portion of the left image are shown in red and green (right). (B) Traces and plots of mean square displacement (MSD) versus time lag (the time between subsequent recordings) for each track of the two individual QD-receptor clusters from panel A. Before ligand addition, the receptor (i) was freely but slowly mobile $\left(0.003 \pm 0.001 \mu \mathrm{m}^{2} / \mathrm{s}\right)$ and became more mobile $\left(0.021 \pm 0.005 \mu \mathrm{m}^{2} / \mathrm{s}\right) 2 \mathrm{~min}$ after addition of $2.5 \mathrm{nM} \mathrm{PTH}$. Zero, 5, and 10 min after PTH addition, the receptor (ii) displayed extremely confined motion $\left(0.0002 \pm 0.0001 \mu \mathrm{m}^{2} / \mathrm{s}, 0.00083 \pm 0.00008 \mu \mathrm{m}^{2} / \mathrm{s}\right.$, and $0.00005 \pm 0.00001 \mu \mathrm{m}^{2} / \mathrm{s}$, respectively). (C) The average motion was determined by probability analysis (see the "Materials and methods" section) and plotted as MSD versus time lag. The motion before PTH addition ( $t=0$ min) is indicated by the solid line with dark circles and after PTH addition by the dotted line with open circles. (D) Four images acquired at 2, 5, 10, and 15 min after ligand application display the internalization behavior for the zoomed image from panel A. (E) Temporal statistics from more than 10 cells for the internalization of ACP-PTHR-TC after addition of $2.5 \mathrm{nM} \mathrm{PTH.} \mathrm{(F)} \mathrm{Trafficking} \mathrm{of} \mathrm{ACP-PTHR-TC} \mathrm{receptor} \mathrm{from} \mathrm{the} \mathrm{membrane} \mathrm{into} \mathrm{the} \mathrm{cell.} \mathrm{Overlay}$ fluorescence and transmission image of four QD-receptor complexes with one trace showing two consecutive steps from the membrane (first image). Zoomed images (right three) of the QD-receptor complex at the membrane in the first image of the sequence with the same trafficked receptor complex (second image) and 2 and 3 min later (third and fourth images). Intensity scaling in panels A and D is in photons $/ 5$ ms.

Clara, CA, USA), and the EMCCD camera system (CascadeII 512B).

Mobility analysis

By plotting the mean square displacement $\left(r_{i}^{2}\right)$ versus $t_{\text {lag }}$, the diffusion behavior of the respective populations of molecules is revealed. The $\left(r_{i}^{2}, t_{\text {lag }}\right)$ data sets were fitted to either the free diffusion model

$$
r_{i}^{2}\left(t_{\text {lag }}\right)=4 D t_{\text {lag }}
$$

[Eq. 1]

the anomalous subdiffusion model

$$
D=\Gamma t_{l a g}^{1-\alpha},
$$

or the confined diffusion model

$$
r_{i}^{2}\left(t_{\text {lag }}\right)=\frac{L^{2}}{3} \times\left[1-\exp \left(\frac{-12 D_{0} t_{\text {lag }}}{L^{2}}\right)\right],
$$

where the mean-square displacement $r_{i}^{2}$ is proportional to time $t_{\text {lag }}, D$ or $\Gamma$ are the diffusion constants, $\alpha$ is the anomalous power lay coefficient, and $L$ is the confinement length (8). For more details about this analysis, see the "Mobility analysis" section of the Supplementary Materials.

\section{Results and discussion}

With the recent advances in general labeling schemes for covalent bonding of both organic dyes and QDs, we were interested in creating a direct and simple QD labeling method using a shorter linkage to improve tracking, internalization, and trafficking studies of single receptors. We devised a technique that uses the well-established system of the ACP as an extracellular fusion protein tag for receptors (30). However, this first required the labeling of QDs with CoA. We used commercially available polymer-coated CdSe/ZnS QDs and straightforward thiol-reactive chemistry with an appropriate concentration of amine blocking groups on the QDs to achieve a maximal 1:1 CoA:QD ratio (Figure 1 and Supplementary Figure S1).
We next tested if the CoA-QDs would specifically and covalently bind to ACP fused to the extracellular domains of receptor proteins upon enzymatic reaction with PPTase (Figure 1). For ACP extracellular receptor fusions, we selected two receptor candidates that are truncation mutants: a truncation of the PTHR (32) and a truncation of the BRII $(33,34)$, called ACP-PTHR-TC and ACP-BRII-TC1, respectively $(33,34)$. ACP-BRII-TC1 resembles the BRII lacking almost the entire cytoplasmic domain, with the extracellular fusion of ACP at the very $\mathrm{N}$ terminus of the truncated receptor. ACP-PTHR-TC resembles the PTH type 1 receptor except that it lacks transmembrane domains III to VII and the long 150-amino acid $\mathrm{C}$ terminus and has the $\mathrm{ACP}$ sandwiched in the $\mathrm{N}$-terminal ligand binding region.

CoA-QD labeling of HEK293 cells transiently transfected with low levels of either ACP-PTHR-TC or ACP-BRII-TC1 marked the receptors with an efficiency and intensity that is well-suited for tracking them on the plasma membrane (Figure 2, A and B). We verified the specificity of the ACP reaction by applying CoA-QDs without the enzyme 
A
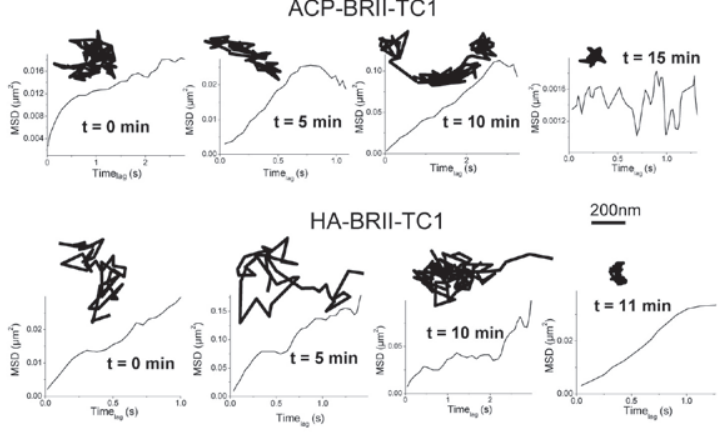

C
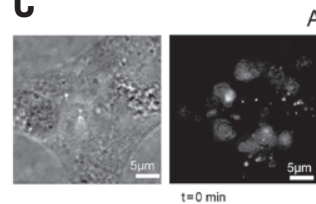

ACP
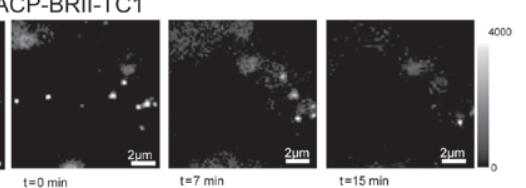

\section{D}

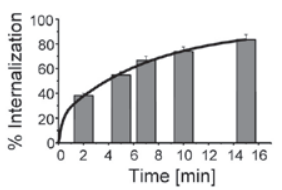

Figure 4. Tracking and internalization of the BRII-TC1 receptor on HEK293 cells. (A) Comparison of tracking from QD-receptors with ACP-CoA-QD labeling (upper) and HA/anti-HA/anti-mouse- $\mathrm{F}_{\mathrm{ab}} \mathrm{-QD}$ labeling (lower) with MSD versus time lag plotted for each track after addition of $1 \mathrm{nM} \mathrm{BMP-2}$. (B) The average motion was determined by probability analysis (see the "Materials and methods" section) and plotted as the MSD versus time lag of all ACP-BMPII-TC1 (top graph) and HA-BMPII-TC1 (lower graph) receptors before (dotted line with open circles) and after (solid line with dark circles) ligand addition. (C) Transmission (first image) and TIRF images (right four) of the CoA-QD ACP-BRIITC1 on HEK293 cells acquired at 0, 7, and 15 min after $1 \mathrm{nM} \mathrm{BMP-2}$ addition (right three magnified images are of the same region at $t=0,7$, and $15 \mathrm{~min}$ ) to display the internalization behavior. Intensity scaling is in photons $/ 5 \mathrm{~ms}$. (D) Temporal statistics from $>10$ cells for the internalization of ACP-BRII-TC1 after 1 nM BMP-2 addition.

PPTase on the same transfected HEK293 cells used in Figure 2, A and B. The CoA-QDs never attached and always fully washed away (Figure 2, A and B). Clearly, the signals came only from the labeled receptors as nonstained cells never displayed the bright spots of the QDs under these conditions and since nontransfected cells never bound CoA-QD even with PPTase treatment (Figure 2C).

We also sought to compare the efficiency of CoA-QD labeling by subjecting the same transfected cells to labeling with antibodyconjugated QDs. As the BRII-TC1 receptor was also available with an extracellular $\mathrm{HA}$ tag $(33,34)$, we compared the HA-tagged receptor stained with mouse anti-HA primary and anti-mouse- $\mathrm{F}_{\mathrm{ab}}-\mathrm{QD}$-conjugated secondary antibody to the CoA-QD-ACP-labeled receptor. We observed that the HA labeling exhibited, on average, the same number of labeled receptors per cell as the ACP-CoAQD method (Figure 2, compare panel D with panel B).

We then tracked the covalently CoA-QDtagged ACP-PTHR-TC on living cells. Although there is a previous report on the diffusion of the whole PTHR receptor (37), it was not certain what type of mobility this extremely truncated receptor might have and what role it might play. Using TIRF microscopy, we imaged the receptors on the basolateral surface of cells (Figure 3A). Upon tracking, we easily identified long (>50 step) traces of individual QD-receptor complexes, primarily indicating free diffusion both before and after PTH addition, followed by quick internalization (Figure 3B, ia and ib). A small subpopulation of QD-receptor complexes displayed restricted mobility both before and after ligand addition and usually endured a long time prior to internalization, if they were internalized at all (Figure 3B, iia-iic). The motion of all QD-receptor complexes was analyzed before and after ligand addition to determine the average motion (Figure 3C). Prior to PTH application, the QD receptor complexes diffused in a partially confined manner on the membrane with a diffusion coefficient of $0.014 \pm 0.002 \mu \mathrm{m}^{2} / \mathrm{s}$ and a confinement coefficient $c=0.739 \pm 0.041$. Slow motion was exhibited by the QD-receptor complexes that remained at the membrane $>10 \mathrm{~min}$ after PTH stimulation; average motion was determined to be just above the ascertained positional accuracy, yielding a value of 0.00033 $\pm 0.00005 \mu \mathrm{m}^{2} / \mathrm{s}$.

Since the tether should be $\sim 1$ order of magnitude shorter for the ACP-CoA-QD labeling in comparison to the anti-HA/antimouse- $\mathrm{F}_{\mathrm{ab}}-\mathrm{QD}$ labeling, we decided to compare the movement of the receptors for any obvious differences. We tracked the ACP-BRII-TC1 and HA-BRII-TC1 on cells both before and after addition of BMP-2. Before and after ligand application, the ACP-BRII-TC1 and HA-BRII-TC1 receptors demonstrated freely diffusing behavior (Figure 4, A and B) until just before the receptors internalized, whereupon the motion either significantly slowed $D_{\text {lat, ACP, } 10 \text { min after }}=0.0004 \pm 0.0002 \mu \mathrm{m}^{2} / \mathrm{s}$ or became confined to regions of $\sim 270 \pm 20 \mathrm{~nm}$ in diameter. However, the diffusion trajectories from the HA-tagged and labeled receptors always appeared to display a larger motion, but this motion did not resultin asignificantlylarger diffusion coefficient of the average mobility behavior (Figure $4 \mathrm{~B}$ ), where before BMP-2 addition $D_{\text {lat, ACP, before }}=0.014 \pm 0.002 \mu \mathrm{m}^{2} / \mathrm{s}$ and $D_{\text {lat, HA, before }}=0.018 \pm 0.004 \mu \mathrm{m}^{2} / \mathrm{s}$.

The internalization behavior for both ACP-QD-labeled receptors was then examined. In each case, the TIRF and widefield images of the basolateral surface of cells were analyzed for instances when a QD-receptor complex would internalize and disappear from the membrane in longer timelapse sequences. An example of each receptor's time-lapse internalization is displayed in Figure 3D for the ACP-PTHR-TC and in Figure $4 \mathrm{C}$ for the ACP-BRII-TC1. Although all four QD-receptor complexes in the example shown for ACP-PTHR-TC (Figure 3D) internalized, on average only $60 \%$ of receptors fully internalized with a single exponential rate of $\sim 0.1$ QD-receptor complex per minute (Figure 3E). The ACP-BRII-TC1 QD-receptors were nearly fully internalized (greater than $80 \%$ ) with apparent bi-exponential rates of 0.1 and 1 QD-receptor complex per minute (80\% and 20\%, respectively; Figure 4D). Recycled QD-receptors appeared in all cases (Figure 4C and Supplementary Figure S2), but the recycled receptors were not included in the determination of internalization rate. The technique is also suitable for longer and larger scale trafficking events by imaging wider fields and increased depths with slightly lower magnification and numerical aperture objectives. Indeed, in many cases, the receptor at the membrane could be tracked for internalization into the cells (Figure 3F).

Here, we demonstrated our labeling technique on decoy versions of the PTH and BRII transmembrane receptors, which each carry the ligand binding site but lack the intracellular domain essential for signaling. The labeling was efficient and specific. Furthermore, the excellent stability of the QDs made it possible to analyze internalization of the receptors with minute timescale time-lapse imaging to detect trafficking of individual receptors from the membrane into the cell and to also observe recycling rates.

The tracking of the receptors with the ACP-QD labeling technique in comparison to antibody-QD labeling displayed slightly less mobility, which we interpret as the effect of the shorter linker length between the receptor and QD. Although we expected high and free diffusion mobility of the truncated receptors, especially in comparison to studies 
performed on their full-length counterparts (37), we observed only moderate but free diffusion. However, before BMP-2 addition, the ACP-BRII-TC1 receptor remained freely diffusive, which was consistent with the observation that it does not bind to other receptors before ligand addition $(33,34,38)$. The incomplete internalization of the ACP-PTHR-TC receptor after ligand addition agrees with previously reported truncation mutants of this receptor (39). The nearly complete internalization of the ACP-BRII-TC1 receptor can be explained by the binding of the receptor to the epidermal growth factor pathway substrate 15 related (EPS15R) protein, a substrate of tyrosine kinase activity of the epidermal growth factor receptor and a constitutive resident in clathrin-coated pits (CCPs), which are the main internalization route of BRII (33). We suggest that binding to EPS15R leads the ligand-bound BRII-TC1 into CCPs, which causes reduced mobility of the receptor, confinement to a region the size of CCPs, and the nearly full internalization of this decoy receptor.

In the future, we expect to show more direct applications with this labeling technique on full-length and specific signaling mutations of membrane receptor proteins to not only track mobility at the membrane, but also to obtain more precise information on internalization dynamics and trafficking. We plan to take advantage of the recently reported 12 -amino acid CoA binding domain $(40,41)$, as well as newly described smaller and biologically benign QDs and nanoparticles $(12,13,18)$, which will make this technique perhaps the smallest, brightest, most specific, and most stable covalent labeling for tracking, internalization, and trafficking studies of individual receptors. In combination with recently described methods for 3-D tracking (42), this would make it possible to determine the entire fate of individual receptors.

\section{Acknowledgments}

We thank Kai Johnsson (École Polytechnique Fédérale de Lausanne [EPFL], Lausanne, Switzerland) for providing us with the ACP and PPTase plasmid constructs. We thank Martin Lohse (University of Würzburg, Würzburg, Germany) and Jean-Pierre Vilardaga (University of Pittsburgh, Pittsburgh, PA, USA) for the N-GFP-PTHR construct, and Asja Guzman (Free University of Berlin, Berlin, Germany) and Mike Friedrich (University of Würzburg) for critically reading the manuscript. This work was supported by the German Science Foundation (grant nos. FZ-82, GK 1048, and 1342 to G.S.H., and grant no. SFB 449, to P.K.) and the Bavarian State Ministry for Research and
Education through the Bio-Imaging Center of the University of Würzburg (G.S.H.).

\section{Competing interests}

The authors declare no competing interests.

\section{References}

1. Alcor, D., G. Gouzer, and A. Triller. 2009. Single-particle tracking methods for the study of membrane receptors dynamics. Eur. J. Neurosci. 30:987-997.

2. Pons, T. and H. Mattoussi. 2009. Investigating biological processes at the single molecule level using luminescent quantum dots. Ann. Biomed. Eng. 37:1934-1959.

3. Chang, Y.P., F. Pinaud, J. Antelman, and S. Weiss. 2008. Tracking bio-molecules in live cells using quantum dots. J. Biophotonics 1:287-298.

4. Kaji, N., M. Tokeshi, and Y. Baba. 2007. Singlemolecule measurements with a single quantum dot. Chem. Rec. 7:295-304.

5. Thompson, R.E., D.R. Larson, and W.W. Webb. 2002. Precise nanometer localization analysis for individual fluorescent probes. Biophys. J. 82:27752783.

6. Kusumi, A., C. Nakada, K. Ritchie, K. Murase, K. Suzuki, H. Murakoshi, R.S. Kasai, J. Kondo, and T. Fujiwara. 2005. Paradigm shift of the plasma membrane concept from the two-dimensional continuum fluid to the partitioned fluid: high-speed single-molecule tracking of membrane molecules. Annu. Rev. Biophys. Biomol. Struct. 34:351-378.

7. Harms, G.S., L. Cognet, P.H. Lommerse, G.A. Blab, H. Kahr, R. Gamsjager, H.P. Spaink, N.M. Soldatov, et al. 2001. Single-molecule imaging of 1-type $\mathrm{Ca}(2+)$ channels in live cells. Biophys. J. 81:2639-2646

8. Lommerse, P.H., G.A. Blab, L. Cognet, G.S. Harms, B.E. Snaar-Jagalska, H.P. Spaink, and T.Schmidt. 2004. Single-molecule imaging of the $\mathrm{H}$-ras membrane-anchor reveals domains in the cytoplasmic leaflet of the cell membrane. Biophys. J. 86:609-616.

9. Jacquier, V., M. Prummer, J.M. Segura, H. Pick, and H. Vogel. 2006. Visualizing odorant receptor trafficking in living cells down to the single-molecule level. Proc. Natl. Acad. Sci. USA 103:14325-14330.

10. Prummer, M., B.H. Meyer, R. Franzini, J.M. Segura, N. George, K. Johnsson, and H. Vogel. 2006. Post-translational covalent labeling reveals heterogeneous mobility of individual $\mathrm{G}$ proteincoupled receptors in living cells. Chembiochem 7:908-911.

11. Sonnleitner, A., L.M. Mannuzzu, S. Terakawa, and E.Y. Isacoff. 2002. Structural rearrangements in single ion channels detected optically in living cells. Proc. Natl. Acad. Sci. USA 99:1275912764.

12. Liu, W., M. Howarth, A.B. Greytak, Y. Zheng, D.G. Nocera, A.Y. Ting, and M.G. Bawendi. 2008. Compact biocompatible quantum dots functionalized for cellular imaging. J. Am. Chem. Soc. 130:1274-1284.

13. Dif, A., F. Boulmedais, M. Pinot, V. Roullier, M. Baudy-Floc'h, F.M. Coquelle, S. Clarke, P. Neveu, et al. 2009. Small and stable peptidic PEGylated quantum dots to target polyhistidinetagged proteins with controlled stoichiometry. J. Am. Chem. Soc. 131:14738-14746. 
14. Curry, A.C., M. Crow, and A. Wax. 2008. Molecular imaging of epidermal growth factor receptor in live cells with refractive index sensitivity using dark-field microspectroscopy and immunotargeted nanoparticles. J. Biomed. Opt. 13:014022.

15. Huang, Y.F., H. Liu, X. Xiong, Y. Chen, and W. Tan. 2009. Nanoparticle-mediated IgE-receptor aggregation and signaling in RBL mast cells. J. Am. Chem. Soc. 131:17328-17334.

16. Wiseman, P.W., P. Hoddelius, N.O. Petersen, and K.E. Magnusson. 1997. Aggregation of PDGF-beta receptors in human skin fibroblasts: characterization by image correlation spectroscopy (ICS). FEBS Lett. 401:43-48.

17. Dorsch, S., K.N. Klotz, S. Engelhardt, M.J. Lohse, and M. Bunemann. 2009. Analysis of receptor oligomerization by FRAP microscopy. Nat. Methods 6:225-230.

18. Howarth, M., W. Liu, S. Puthenveetil, Y.Zheng, L.F. Marshall, M.M. Schmidt, K.D. Wittrup, M.G. Bawendi, and A.Y. Ting. 2008. Monovalent, reduced-size quantum dots for imaging receptors on living cells. Nat. Methods 5:397-399.

19. Howarth, M. and A.Y. Ting. 2008. Imaging proteins in live mammalian cells with biotin ligase and monovalent streptavidin. Nat. Protocols 3:534-545.

20.Zhang, Y., M.K. So, A.M. Loening, H. Yao, S.S. Gambhir, and J. Rao. 2006. HaloTag protein-mediated site-specific conjugation of bioluminescent proteins to quantum dots. Angew. Chem. Int. Ed. Engl. 45:4936-4940.

21.Zhang, Y., M.K. So, and J. Rao. 2006. Protease-modulated cellular uptake of quantum dots. Nano Lett. 6:1988-1992.

22. Griffin, B.A., S.R. Adams, and R.Y. Tsien. 1998. Specific covalent labeling of recombinant protein molecules inside live cells. Science 281:269-272.

23. O'Hare, H.M., K. Johnsson, and A. Gautier. 2007. Chemical probes shed light on protein function. Curr. Opin. Struct. Biol. 17:488-494.

24.Yano, Y. and K. Matsuzaki. 2009. Tag-probe labeling methods for live-cell imaging of membrane proteins. Biochim. Biophys. Acta 1788:2124-2131.

25. Liu, R., D. Hu, X. Tan, and H.P. Lu. 2006. Revealing two-state protein-protein interactions of calmodulin by single-molecule spectroscopy.J. Am. Chem. Soc. 128:10034-10042.

26. Roullier, V., S. Clarke, C. You, F. Pinaud, G.G. Gouzer, D. Schaible, V. Marchi-Artzner, J. Piehler, and M. Dahan. 2009. High-affinity labeling and tracking of individual histidinetagged proteins in live cells using $\mathrm{Ni}^{2+}$ trisnitrilotriacetic acid quantum dot conjugates. Nano Lett. 9:1228-1234.

27. Guignet, E.G., J.M. Segura, R. Hovius, and H. Vogel. 2007. Repetitive reversible labeling of proteins at polyhistidine sequences for single-molecule imaging in live cells. Chemphyschem 8:1221-1227.

28. Chen, I., M. Howarth, W. Lin, and A.Y. Ting. 2005. Site-specific labeling of cell surface proteins with biophysical probes using biotin ligase. Nat. Methods 2:99-104.

29. Lin, C.W. and A.Y. Ting. 2006. Transglutaminase-catalyzed site-specific conjugation of small-molecule probes to proteins in vitro and on the surface of living cells. J. Am. Chem. Soc. 128:4542-4543.

30. George, N., H. Pick, H. Vogel, N. Johnsson, and K. Johnsson. 2004. Specific labeling of cell surface proteins with chemi- cally diverse compounds. J. Am. Chem. Soc. 126:8896-8897.

31. Kropf, M., G. Rey, L. Glauser, K. Kulangara, K. Johnsson, and H. Hirling. 2008. Subunitspecific surface mobility of differentially labeled AMPA receptor subunits. Eur. J. Cell Biol. 87:763-778.

32. Castro, M., V.O. Nikolaev, D. Palm, M.J. Lohse, and J.P. Vilardaga. 2005. Turn-on switch in parathyroid hormone receptor by a two-step parathyroid hormone binding mechanism. Proc. Natl. Acad. Sci. USA 102:16084-16089.

33. Hartung, A., K. Bitton-Worms, M.M. Rechtman, V. Wenzel, J.H. Boergermann, S. Hassel, Y.I. Henis, and P. Knaus. 2006. Different routes of bone morphogenic protein (BMP) receptor endocytosis influence BMP signaling. Mol. Cell. Biol. 26:7791-7805.

34. Nohe, A., S. Hassel, M. Ehrlich, F. Neubauer, W. Sebald, Y.I. Henis, and P. Knaus. 2002. The mode of bone morphogenetic protein (BMP) receptor oligomerization determines different BMP-2 signaling pathways. J. Biol. Chem. 277:5330-5338.

35. Steinmeyer, R., A. Noskov, C. Krasel, I. Weber, C. Dees, and G.S. Harms. 2005. Improved fluorescent proteins for singlemolecule research in molecular tracking and co-localization. J. Fluoresc. 15:707-721.

36. Michel, R., R. Steinmeyer, M. Falk, and G.S. Harms. 2007. A new detection algorithm for image analysis of single, fluorescence-labeled proteins in living cells. Microsc. Res. Tech. 70:763-770.

37. Wheeler, D., W.B. Sneddon, B. Wang, P.A. Friedman, and G. Romero. 2007. NHERF-1 and the cytoskeleton regulate the traffic and membrane dynamics of $\mathrm{G}$ protein-coupled receptors. J. Biol. Chem. 282:25076-25087.

38.Sieber, C., J. Kopf, C. Hiepen, and P. Knaus. 2009. Recent advances in BMP receptor signaling. Cytokine Growth Factor Rev. 20:343355.

39. Vilardaga, J.P., C. Krasel, S. Chauvin, T. Bambino, M.J. Lohse, and R.A. Nissenson. 2002. Internalization determinants of the parathyroid hormone receptor differentially regulate beta-arrestin/receptor association. J. Biol. Chem. 277:8121-8129.

40. Yin, J., P.D. Straight, S.M. McLoughlin, Z. Zhou, A.J. Lin, D.E. Golan, N.L. Kelleher, R. Kolter, and C.T. Walsh. 2005. Genetically encoded short peptide tag for versatile protein labeling by Sfp phosphopantetheinyl transferase. Proc. Natl. Acad. Sci. USA 102:1581515820

41.Zhou, Z., P. Cironi, A.J. Lin, Y. Xu, S. Hrvatin, D.E. Golan, P.A. Silver, C.T. Walsh, and J. Yin. 2007. Genetically encoded short peptide tags for orthogonal protein labeling by Sfp and AcpS phosphopantetheinyl transferases. ACS Chem. Biol. 2:337-346.

42.Katayama, Y., O. Burkacky, M. Meyer, C. Brauchle, E. Gratton, and D.C. Lamb. 2009. Real-time nanomicroscopy via three-dimensional single-particle tracking. Chemphyschem 10:2458-2464.

Received 1 April 2010; accepted 18 June 2010.

Address correspondence to Gregory Harms, Bio-Imaging Center, Rudolf Virchow Center, University of Würzburg, Josef-Schneider-Str. 2, Haus D15, D-97080, Würzburg, Germany. e-mail: gregory.harms@virchow.uni-wuerzburg.de

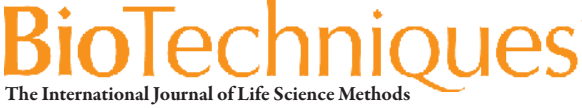

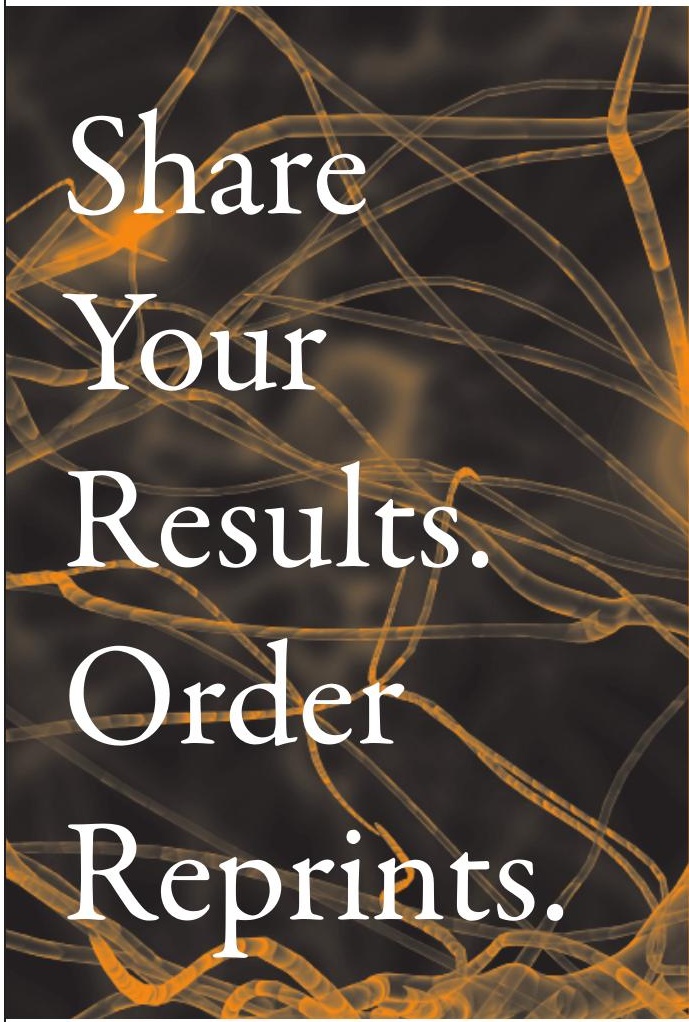

Author Reprints

Make the most of your hard work by ordering reprints of your article published in BioTechniques. Reprints are an inexpensive and easy way to distribute your findings to students and colleagues alike.

\section{Corporate Reprints}

Leverage BioTechniques, the most powerful brand in the market. Reprints help support your sales effort by utilizing articles that spotlight your brand/products to educate customers at meetings and industry trade events.

\section{More info:}

www.BioTechniques.com/reprints 